\title{
The American Pediatric Society MEMBERSHIP LIST* 1976-1977
}

\section{Emeritus}

Adams, John M., 405 Hilgard Avenue, Los Angeles, California 90024

Anderson, John A., M.D., Department of Pediatrics, 1460 Mayo, University of Minnesota, Minneapolis, Minnesota, 55108

Anderson, W. S., M.D., 5300 Westbard Avenue, Washington, D.C. 20016

Arena, Jay M., M.D., Duke Medical Center, Box 3024, Durham, North Carolina 27710

Bain, Katherine, M.D., 2710 Quebec Street N.W. Washington, D.C. 20008

Barba, S. Philip, M.D., 120 Erdenheim Road, Philadelphia, Pennsylvania 19118

Baty, M., James, M.D., 345 March St., Belmont, Massachusetts 02178

Baumgartner, Leona, M.D., 25 Shattuck Sereet, Boston, Massachusetts 02115

Berenberg, Wm., M.D., Harvard Medical School, 300 Longwood Ave., Boston, Massachusetts 02115

Blattner, Russel J., M.D., Baylor College of Medicine, 1200 Moursand, Houston, Texas 77030

Bosma, James F., M.D., Oral and Pharyngeal Development Section, National Institute of Denta Research, Department of Health, Education and Welfare, Bethesda, Maryland 20014

Boyd, Edith, M.D., 4200 East 9th Avenue, Denver, Colorado 80220

Boyd, Julian D., M.D., 1234 Cypress Avenue. Santa Ana, California 92707

Bradford, William L., M.D., 260 Crittenden Blvd., Rochester, New York 14620

Bradley, Charles, M.D., 12397 S.W. King George Tigard, Oregon 97223

Bradley, J. Edmund, M.D., 17842 Sintonte Drive, San Diego,California 92128

Breese, B. B., M.D., 3054 S. Clinton Avenue, Rochester, New York 14623

Bruton, Ogden C., M.D., 13227 Betty Lane, Silver Spring, Maryland 20904

Buchanan, Douglas N., M.D., 920 East 59th Street, Chicago, Illinois 60637

Butler, Allan M., M.D., Tashmoo Farm, Vineyard Haven, Massachusetts 02568

Byers, Randolph K., M.D., 300 Longwood Avenue, Boston, Massachusetts 02115

Caffey, John, M.D., Children's Hospital, 125 DeSoto Street, Pittsburgh, Pennsylvania 15213

Carson, Merl J., M.D., 1771 Sirrine Drive, Santa Ana, California 92705
Cassels, Donald E., M.D., Bobs Roberts Memorial Hospital, 920 East 59th Street, Chicago, Illinois 60637

Castellanos, A., Sr., M.D., Varicty Children's Hospital, 6125 S.W. 31 st Street, Miami, Florida 33155

Chapple, Charles C., M.D., 7307 Eby Drive, Shawnee Mission, Kansas 66204

Chipman, Sidney, M.D., Rogers Hill Road, Bradford, Vermont 05033

Chown, Bruce, M.D., 735 Notre Dame Avenue, Winnipeg 3, Manitoba, Canada

Christie, Amos U., M.D., Vanderbilt University, School of Medicine, Nashville, Tennessee 37203

Christopherson, E. H., M.D., 222 Quince Street. Apt. 3C, San Diego, California 92103

Clifford, Stewart H., M.D., 33 Water Street, Box 278, Duxbury, Massachusetts 02332

Crump, E. Perry, M.D., Meharry Medical College, School of Medicine, Department of Pediatrics. Nashville, Tennessee 37208

Curnan, Edward C., Jr., M.D., 630 West 168th Street, New York, New York 10032

Davies, John A. V., M.D., 235 Sargent Road, Brookline, Massachusetts 02146

Day, Richard L., M.D., Lakeview Terrace, Westbrook, Connecticut 06498

Deamer, William C., M.D., 3 Fifth Avenue, San Francisco, California 94118

Dees, Susan C., M.D., Box 2913, Duke University Medical Center, Durham, North Carolina 27710

Denton, Ronald Lyman, M.D., R. 1, North Hatley, Quebec, Canada

Diamond, L. K., M.D., University of California Medical Center, San Francisco, California 91422

Dietrich, Harry F., M.D., Camarillo State Hospital, Box A, Camarillo, California 93010

Dushane, J. W., M.D., Mayo Clinic, 200 First Street S.W., Rochester, Minnesota 55902

Ebbs, J. H., M.D., 50 Prince Arthur Avenue, Suite 803 , Toronto, Canada

Eliot, Martha M., M.D., 21 Francis Avenue, Cambridge, Massachusetts 02138

Faber, Harold K., M.D., 2351 Clay Street, San Francisco, California 94115

Farr, Lee E., M.D., 1940 Broadway, San Francisco, California 94109

Fashena, Gladys J., M.D., University of Texas, Southwestern Medical School, 5223 Harry Hines Blvd., Dallas, Texas 75235
Felton, Harriet H., M.D., 8923 Wickfield, San Antonio, TX 78217

Fraad, Lewis M., M.D., Bronx Municipal Hospital Center, Pelham Parkway and Eastchester Road, Bronx, New York 10461

Gallagher, J. R., M.D., 67 Millrock Road, Hamden, Connecticut 06511

Gitlin, David, M.D., Children's Hospital of Pittsburgh, 125 DeSoto, Pittsburgh, Pennsylvania 15213

Goettsch, Elvira, M.D., 183 Third Avenue Apartment 604, Chula Vista, California 92010

Gordon, Harry H., M.D., 1300 Morris Park Avenue, New York, New York 10461

Guild, Harriet G., M.D., 601 North Broadway, Baltimore, Maryland 21205

Hamilton, Bengt K., M.D., 110 LaPaloma Street, Santa Fe, New Mexico 87501

Harper, Paul, M.D., 615 North Wolfe Street, Baltiriore, Maryland 21205

Harris, Jerome S., M.D., Duke University, Durham, North Carolina 27706

Harrison, Harold E., M.D., 5500 N. Charles Street, Baltimore, Maryland 21210

Heymann, Walter, M.D., 2103 Adelbert Road, Cleveland, Ohio 44106

Hill, Lee Forrest, M.D., 3232 John Lynde Road, Des Moines, lowa 50312

Hodes, Horace L., M.D., Mt. Sinai School of Medicine, Department of Pediatrics, Fifth Avenue and 100th Street, New York, New York 10029

Hubbard, John P., M.D., 3930 Chestnut Street, Philadelphia, Pennsylvania 19104

Jackson, Edith B., M.D., 4200 East Ninth Avenue, Denver, Colorado 80220

Jackson, Robert L., M.D., University of Missouri, School of Medicine, Department of Pediatrics, Columbia, Missouri 65201

Janeway, Charles A., M.D., Children's Hospital Medical Center, 300 Longwood Avenue, Boston, Massachusetts 02115

Johnston, Joseph A., M.D., 1937 Boston Boulevard, Detroit, Michigan 48206

Josephs, Hugh Wilson, M.D., 915 Rolandvue Road, Ruxton, Maryland 21204

Karelitz, Samuel, M.D., The L. I. Jewish Hospital, 270-05 Seventh-Sixth Avenue, New Hyde Park, Long Island New York 11040

Katsampes, Chris P., M.D., 48 Holly Drive, Short Hills, New Jersey 07076

* The Emeritus and Honorary Membership lists of the American Pediatric Society were inadvertently omitted in the April Program issue of Pediatric Research where they customarily appear. 


\section{APS Emeritus}

Keith, Haddow M., M.D., Mayo Clinic, 924 9th Avenue S.W., Rochester, Minnesota 55902

Kunstadter, Ralph H., M.D., P. O. Box 808 Christiansted, Saint Croix, Virgin Islands 00820

Langford, William S., M.D., 120 Carbrini Boulevard, New York, New York 10033

Lesser, Arthur J., M.D., 2924 Rittenhouse Street N.W., Washington, D. C. 20015

Lichty, John A., M.D., 90 South Jersey Street, Denver, Colorado 80222

Lincoln, Edith, M.D., Wallace Road, North Salem, New York 10560

Logan, George B., M.D., 1115 Plummer Circle, Rochester, Minnesota 55901

Lourie, Reginald S., M.D., 4305 Thornapple Street, Chevy Chase, Maryland 20015

Lyon, George M., M.D., 1400 Dale Lane, Delray Beach, Florida 33444

Lyon, Robert A., M.D., University of Cincinnati Medical School, Cincinnati, Ohio 45219

McBryde, Angus M., M.D., Duke University, School of Medicine, Durham, North Carolina 27706

McCarty, Maclyn, M.D., Rockefeller Institute, New York, New York 10021

McCreary, John F., M.D., Faculty of Medicine, University of British Columbia, Vancouver 8, British Columbia, Canada

McCune, Donovan J., M.D., Permanente Hospital, 2600 Alameda Street, Vallejo, California 94590

McGuinnesse, Aims C., M.D., 721 Prospect Avenue, Princeton, New Jersey 08540

McIntosh, Rustin, M.D., Tyringham, Massachusetts 01264

McKhann, Charles F., M.D., 485 Park Avenue, New York, New York 10022

May, Charles D., M.D., National Jewish Hospital 3800 East Colfax Avenue, Denver, Colorado 80206

Medovy, Harry, M.D., Children's Hospital, Winnipeg, Manitoba, Canada

Merritt, Katharine K., M.D., 439 East 51 st Stre New York, New York 10022

Miller, Herbert C., M.D., Department of Pediatrics, University of Kansas Medical Center, 39th Street at Rainbow Boulevard. Kansas City, Kansas 66103

Najjar, Victor A., M.D., Tufts University School of Medicine, 136 Harrison Avenue, Boston, Massachusetts 02111

Nelson, Waldo E., M.D., 615 Moreno Road, Narberth, Pennsylvania 19072
Nemir, Rosa Lee, M.D., New York University School of Medicine, 550 First Avenue, New York, New York 10016

Neuhauser, Edward B. D., M.D., 300 Longwood Avenue, Boston Massachusetts 02115

Ortiz, Antonio, M.D., University of Puerto Rico, School of Medicine, San Juan, Puerto Rico 00905

Peterson, J. C., M.D., Medical College of Wisconsin, 1700 West Wisconsin Avenue, Milwaukee, Wisconsin 53233

Pierce, Mila I., M.D., 5801 South Dorchester, Chicago, Illinois 60637

Pincus, Joseph, M.D., 881 Washington Avenue, Brooklyn, New York 11225

Potter, Edith L., M.D., Route 3, Box 658, Fort Myers, Florida 33901

Ross, Alan, M.D., 77 Windsor Avenue, Westmount, Montreal, Quebec, Canada

Ross, S. Graham, M.D., 65 Rosemont Crescent, Westmount, Providence of Quebec, Canada

Rubin, Mitchell I., M.D., 22 Charlestowne Court, Charleston, South Carolina 29401

Sabin, Albert B., M.D., Medical University of South Carolina, 80 Barre Street, Charleston, South Carolina 29405

Sanford, Heyworth N., M.D., 650 Orange Street, New Haven, Connecticut 0651

Schaffer, Alexander J., M.D., 1190 W. Northern Parkway, Baltimore, Maryland 21210

Schmidt, William M., M.D., c/o Harvard School of Public Health, 55 Shattuck Street, Boston, Massachusetts 02115

Scott, Roland B., M.D., Freedmen's Hospital, Department of Pediatrics, Washington, D.C. 2000

Scott, Thomas F. M., M.D., Department of Pediatrics, Hahnemann Medical College-Hospital, 230 North Broad Street, Philadelphia, Pennsylvania 19102

Seelye, Walter B., M.D., 2264 Del Norte, Los Osos, California 93402

Senn, Milton J. E., M.D., 333 Cedar Street. New Haven, Connecticut 06510

Shaffer, Thomas E., M.D., 3868 Chevington Road, Columbus, Ohio 43220

Shaw, Edward B., M.D., 65 Arguello Boulevard, San Francisco, California 94118

Silverthorne, L. Nelles, M.D., 8 Brendan Road, Toronto 17, Ontario, Canada

Smith, Clement A., M.D., 300 Longwood Avenue, Boston, Massachusetts 02115

Smith, Richard M., M.D., 401 Beacon Street, Boston, Massachusetts 02116
Spock, Benjamin M., M.D., 538 Madison Avenue, New York, New York 10022

Stearns, Genevieve, M.D., Children's Hospital, lowa City, Iowa 52240

Stevenson, Stuart S., M.D., St. Luke's Hospital Center, Amsterdam Avenue at 114th Street. New York, New York 10025

Struthers, Robert R., M.D., c/o Bank of Montreal 600 University Avenue, Toronto 2, Ontario Canada

Stuart, Harold C., M.D., 55 Shattuck Street, Boston, Massachusetts 02115

Swanson, William W., M.D., 9515 Raintree Drive, Sun City, Arizona 85351

Taussig, Helen B., M.D., The Johns Hopkins Hospital, Baltimore, Maryland 21205

Thelander, Hulda E., M.D., 40 Seafirth Place Tiburn, California 94920

Ward, Robert, M.D., 846 Paseo Miramar, Pacific Palisades, California 90272

Warkany, Josef, M.D., Children's Hospital, Cincinnati, Ohio 45229

Webb, Clarence H., M.D., The Children's Clinic, 1560 Line, Shreveport, Los Angeles 71101

Weech, A. Ashley, M.D., Department of Pediatrics, University of Florida, College of Medicine. Gainesville, Florida 32610

Wegman, Myron E., M.D., School of Public Health, University of Michigan, University of Michigan, Ann Arbor, Michigan 48104

Wetzel, Norman C., M.D., 2358 Ardleigh Drive, Cleveland Heights, Ohio 44106

Weymuller, Charles, M.D., 1021 Adams Street S.E., Albuquerque, Northern Maine 87108

Wheatley, George M., M.D., Metropolitan Life Insurance Company, One Madison Avenue, New York, New York 10010

Wilson, James L., M.D., University Hospital Ann Arbor, Michigan 48104

Wolman, Irving J., M.D., 7607 Woodlawn Avenue, Melrose Park, Pennsylvania 19126

Woolley, Paul V., Jr., M.D., Professor of Pediatrics, Wayne State University, Children's Hospital of Michigan, 3901 Beaubien, Detroit, Michigan 48201

Wright, F. Howell, M.D., 5739 Kimbark Avenue, Chicago, Illinois 60637

Wyman, Edwin T., M.D., Sebec, Maine 0448

Yannet, Herman, M.D., Heritage Village 523-B, Southbury, Connecticut 06488

Zellweger, Hans U., M.D., Department of Pediatrics, University Hospitals, Iowa City, Iowa 52242

Zuelzer, Wolf W., M.D., 1251 Cresthaven Drive, Silver Spring, Maryland 20903 